\title{
REVISIÓN DE REVISTAS
}

\author{
POR \\ ALEX DE ALAVA \\ Universidad de la República, Montevideo
}

non novus, sed nove.

Las incomodidades de las reseñas no tienen límites. En el mejor de los casos, el articulista incursiona en un hábito que intenta negar, su flechada predilección, sus olvidos a deshora, su método adivinatorio, con una parrafada de nombres, números, manifiestos, pasiones.

Así, a las revistas de los últimos cuarenta años les urgen casi los mismos designios que a sus anteriores. Periódicamente, y desafiando su heredado estigma, aparecen en Montevideo y en el interior del país, nuevas revistas que luchan contra lo ineluctable: perecer antes del tercer número editado.

La historia de estos cambios y permanencias es también la entonación diversa de una misma metáfora: llevar un hecho individual a la vida colectiva. Publicarlo. "Venderlo en subasta pública."

Para ello deberíamos entender el signo de la revista que se desdobla en un mismo plano. Por un lado, como síntesis del hecho literario que se ofrece al mundo, por otro lado, como hecho literario en sí, que se da a sí mismo. Ahora bien, cada una de ellas, la revista literaria y la literatura, marca y enmascara a la otra, la legitima y la compensa. Son dos caras y una de ellas no es siempre el significado, y la otra, el significante. Más bien parecería que ambas se significan recíprocamente. Según el punto de vista metodológico de la pesquisa, cada una de ella es significante y significado.

Entonces cada lector leerá a su manera, situará, según sus gustos, lo que leyó en lo concreto o en lo abstracto, en lo pragmático o en el sueño. Soñará lo que vio y verá aquello que estaba soñando.

La revista, como obra literaria, apela a la misma retórica que la escritura publicitaria; tiene la misma función metafórica: hacer apasionante (sin pasión) aquello que carece de interés.

Esto sin tener demasiado en cuenta que el país de tres millones de hombres, posee una literatura hecha a la escala del centenar. Esta afirmación no intenta

\footnotetext{
${ }^{1}$ Citado por Robert Escarpit: el empleo más antiguo de la palabra publicare, que cita Littré, data del siglo XIII y, aplicado a bienes muebles, significa "vender en subasta pública".
} 
despertar la polémica, sino conducir a la reflexión. Estimular nuevas investigaciones y descubrimientos, considerar y reconsiderar la industria editorial literaria, sus funciones y su lugar.

Comprenderá pues el lector que no tiene delante una guía de revistas de los últimos cuarenta años; tarea casi imposible dado que las mismas sobrepasan largamente el centenar, y no es propósi to de quien esto escribe hacer un tratado minucioso y democrático de lo editado entre 1950 y 1990.

Sí, nos falta, para terminar esta introducción, presentar excusas. Es por demás obvioque quedarán por el camino nombres, títulos, preguntas importantes. A cambio intentaremos mostrar la eficacia, la naturaleza y la extensión de influencia de algunas revistas, lo aperiódico de otras, la persistencia unipersonal en lo editorial, el aporte a la cultura nacional, sus propuestas y nuestra tarea crítica. No se trata de practicarla a nivel de las respuestas (como lo aconsejan las buenas costumbres y la generación del 45) sino de ejercitarla también a través de las preguntas. Y esta crítica no es tarea de nadie en especial, sino el derecho de todo lector avisado.

\section{NOMBRES}

I. Resulta inimaginable cualquier estudio de nuestras revistas sin mencionar los nombres de Asir y Número. No son arquetipo ni panacea de nada, pero sí marcan con precisión los dos grupos de los que se compuso la que se llama "generación del cuarenta y cinco", en el supuesto de que esa generación haya existido.

Asir marca una dirección que estuvo fundamentalmente centrada en la preocupación de la realidad uruguaya, sobre todo la realidad literaria. Número tiene un aspecto más universal, señalado por la influencia de la literatura inglesa, francesa y norteamericana. Sin embargo, como ocurre frecuentemente, tanto una como otra revista tuvieron en el transcurso de su vida editorial sus interinfluencias.

Hay en Asir dos períodos bien señalados. El iniciado en Mercedes bajo la dirección de Washington Lockhart - " con una tendencia provinciana a decir de sus detractores" - hasta el número siete; para luego tomar una físonomía muy marcada por la presencia de Domingo Bordoli y colaboradores de la talla de Líber Falco, Guido Castillo, Dionisio Trillo Pais, Arturo Sergio Visca. La nueva revista se juega por una revista uruguaya que tenga la vista fijada en nuestra realidad. "Con rostro uruguayo, no parisino." Es por esa época que aparecen nuevas sesiones, como "Literatura ejemplar", donde se estudiaban problemas literarios con "ejemplos" comentados. En más de una oportunidsd se acusó a Asir por tener una predilección casi exclusiva por la narrativa campera, cosa que

\footnotetext{
${ }^{2}$ Entrevista mantenida con Arturo Sergio Visca.

${ }^{3}$ Entrevista mantenida con Arturo Sergio Visca.
} 
no es exacta. La literatura es campera o ciudadana, fantástica o realista, pero lo primero que importa es si es literatura. Es verdad que Asir recoge muchos autores camperos, pero a más de treinta años de editada es dable observar que la literatura campera estaba en auge, y el crítico analizaba la tendencia que se daba en su momento. "Asir fue una revista que tuvo significación por dos cosas. Primero porque se orientó de acuerdo con criterios bien definidos, no fue una mera reunión azarosa de unas cuantas personas, sino que estuvo regida por un grupo bastante homogéneo en sus concepciones generales de la literatura, aunque cada uno tenía sus gustos a veces coincidentes a veces discrepantes ... y segundo porque sirvió para fijar una postura con respecto a la literatura nacional, que le abrió camino a varios escritores y poetas, y que saca una cantidad de números donde hay material de real interés" ${ }^{4}$

Como editorial, Asir publicó dos libros de cuentos de Julio C. Da Rosa, dos novelas y tres libros de poesía, entre ellos El Poeta de W. Benavídez, y Tiempo y Tiempo de Líber Falco.

Número, a pesar de su ya señalada tendencia universal, dedica un número íntegro a nuestra generación del novecientos (Asir respondió con uno dedicado a José Martí en el centenario de su nacimiento), con lo cual queda demostrado que sus intereses trascendían la imagen que cada uno se había hecho del otro. Como editorial, Número publicó Aspectos de la literatura gauchesca de Jorge Luis Borges; Quién de nosotros y Solo mientras tanto de Mario Benedetti;El rapto y otros cuentos de Francisco Espínola, y libros de poesía donde figuran Juan Cunha, Humberto Megget e Idea Vilariño.

Para terminar con la reseña de estas dos prestigiosas revistas, decir unas palabras de la falsa confrontación en que se las puso. Cuando se habla de la generación del 45 se tiene puesta la vista en Marcha y en la gente que salió de ella: Rodríguez Monegal, Martínez Moreno, Rama e Idea Vilariño en poesía; menos considerando a gente de la estatura de Domingo Bordoli, Guido Castillo, Arturo Sergio Visca. Todos estos nombres pertenecen a una misma generación pero a grupos distintos que crean las conocidas luchas intrageneracionales. $\mathrm{Si}$ estudiáramos la historia de la cultura uruguaya en general, no podríamos evitar la mención de Marcha en todas sus épocas y descubriríamos que en diferentes períodos todos estos nombres fueron colaboradores del acreditado semanario.

Hacia el final de su vida editorial y por iniciativa del escritor Arturo Sergio Visca, ambas revistas se unen para formar "Ediciones de Asir". Aparecerán títulos como El país de la cola de paja de Mario Benedetti; Las raíces de Horacio Quiroga de Emir Rodríguez Monegal; Tierra sin mapa de Ángel Rama; $E l$ patriciado uruguayo de Carlos Real de Azúa; El mundo no es absurdo de Washington Lockhart; El infierno tan temido de Juan Carlos Onetti y Sendero Solo de Domingo Luis Bordoli.

${ }^{4}$ Entrevista mantenida con Arturo Sergio Visca. 
II. Existieron dos importantes publicaciones, que a la jerarquía de sus trabajos, aportaron la edición bilingüe español-francés.

La Licorne fue una revista fundada en el año 1947 por la inolvidable Susana Soca. Sus primeros números se editaron en París, y a partir del número siete, en 1953, se editó en Montevideo, en español y francés con el nombre de Entregas de La Licorne. Esta revista publicó por primera vez en español un trabajo de Boris Pasternak, antes de que apareciera en ruso. Incluso se cuenta (y esto puede formar parte del mito) que tuvo la posibilidad de publicar Dr. Zhivago en el Uruguay antes de que fuera publicado en ningún otro idioma.

Por su ascendencia francesa, Entregas de La Licorne es una revista metida en el Uruguay pero proveniente de otro lado. Susana Soca fue una persona que se formó en el extranjero, y de su muchísima experiencia guardó destrezas, inesperados sesgos, amigos repentinos, que marcaron lo singular de la revista.

Borges, Bioy Casares, Bordoli, Alberti, Roberto Ibáñez, Jorge Guillén, Juana de Ibarbourou, Felisberto Hernández, Alberto Moravia, Pablo Neruda, Karl Jaspers, Silvina Ocampo, Zum Felde, Vaz Ferreira, Real de Azúa, Jules Supervielle, Clara Silva, Alfonso Reyes, fueron algunos de sus colaboradores.

El último número ( $\mathrm{n}^{\mathbf{0}}$ 16) fue publicado luego de la muerte de su fundadora, y dirigido por Guido Castillo. En la reseña del libro El costado del fuego de Ricardo Paseyro, el propio Castillo dice textualmente que se está ante "uno de los mejores libros de poesía escritos en lengua española de estos últimos años”.

La revista le dio mucha importancia a lo español y para ello contó con la colaboración permanente del gran José Bergamín.

La otra revista “anfibia" es Maldoror. Su primer número apareció en el año 1967 y hasta su número 9 fue bilingüe. Más allá de la similitud con La Licorne en la bilingualidad, Maldoror expresa una de las caras de la realidad uruguaya: la influencia muy fuerte de la cultura francesa en nuestro país. Hay una marcada preocupación (sobre todo en sus últimos números) por realizar una actualización teórica. A partir del restablecimiento de la democracia, dirigido a un público de estudiosos de literatura que, durante los doce años de gobierno militar permaneció aislado y alejado de las principales corrientes de la teoría literaria, el $n^{0} 19$ de Maldoror aparece dedicado enteramente a las teorías de la recepción literaria y de la experiencia estética, el n” 20 a "El texto según Gérard Genette" y el n²1 a "Jacques Derrida: Primeras (pre)ferencias". Bajo el sello "Ediciones Maldoror" publicó dos libros de poemas: Ápices de José García Rey y Situación anómala de Jorge Medina Vidal, y dos partituras Meditación en fa de Juan José Iturriberry y Ecos de Héctor Tosar. Con el sello “x y z”, en 1987, editó Diseminario: la desconstrucción, otro descubrimiento de América, publicación coordinada, de la misma manera que los números de teoría, por Lisa Block de Behar.

5 Término empleado por Arturo Sergio Visca. 
III. En el año 1929 se publica por primera vez en Montevideo el número 61 de la revista Alfar.$^{\circ}$ Este párrafo está dedicado a la memoria de su director Julio C. Casal. Por su esfuerzo personal la revista siguió saliendo hasta su número 91 (marzo de 1955). Por sus páginas pasaron varias generaciones de notorios hombres de letras cuya nominación se hace imposible.

IV. En el otoño de 1963 aparece el primer número de la revista Puente, "revista de letras radicada en Uruguay y abierta a las letras de Israel". En sus más de 190 páginas encontramos poemas de Circe Maia, Idea Vilariño, Ricardo Paseyro, fragmentos de Don Juan el Zorro de F. Espínola (que ya habían aparecido en Asir), ensayos de M. Benedetti y E. Rodríguez Monegal, tres escritos de Martín Buber, y un notable trabajo de Erza Heymann sobre "El significado antropológico del lenguaje”. La diagramación y presentación de la revista era muy original, y la impresión una suerte de palimpsesto en diversos tipos de papel impresos sobre láminas pre-impresas.

En junio de 1982, aparece el primer número de UNO en la cultura. Con más de veinte páginas destinadas a difundir la poesía de Juan Gelman, la revista intentó mostrar un perfil. Hay un reportaje a Belchior (músico brasileño, alumno de Haroldo de Campos), trabajos sobre teatro, títeres, una encuesta a músicos uruguayos y una notoria juventud en todos los integrantes de su consejo editor. Tiraron quinientos ejemplares, y los vendieron uno por uno. En la confección gráfica de la revista lo más notorio fue el destierro de "helvéticas y bodonis" por tipos menos vistos.

Puente y UNO en la cultura nunca editaron su número dos. No es nuestra intención al mencionarlas hacer un culto de lo efímero (de eso se encarga nuestra época) sino de recordar sin nombrarlas, las casi 30 publicaciones que corrieron la misma suerte. De todas formas, cada una de ellas fue única a su manera, la manera mejor y más hermosa de ser única.

V. El año 1950 no sólo marca el límite inicial de nuestro trabajo, también es el año en que deja de publicarse la revista con mayor cantidad de números editados en nuestro país (172). Su nombre: HIPERION.

\section{NÚMEROS}

Nuestra época quiere que todo sea público, que nada quede sin medirse. El animal totémico pretende que nada sea secreto, que todo se cuente. Se cuenta lo que nadie vio y se cuenta la actualización de lo que nadie vio. Con más razón la cosa escrita, que en su recurrencia permite casi instantáneamente volver atrás. Hacer una, dos, tres, veinte lecturas si es preciso. Si pactamos con

\footnotetext{
${ }^{6}$ Alfar fue una publicación española, nacida en octubre de 1923, en La Coruña.
} 
nuestra época, aparece la cifra, el porcentaje, la estadística, el valor de cambio del signo, y cuatro períodos para una mejor referencia.

I. Entre 1950 y 1960 se publicaron en nuestro país cuarenta y tres revistas, siendo las más significativas las ya mencionadas Asir, Número, La Licorne y Alfar. Catorce revistas no llegaron a editar su tercer número.

II. Entre 1960 y 1972 se publicaron 38 revistas. Aparece Maldoror que se publicará hasta 1985 discontinuamente y con una nuevainterrupción reaparecerá sólo un número en 1987. Y también Aqui Poesía, Los Huevos del Plata, Monte Sexto y Puente. Siete revistas publicaron un sólo número.

III. Entre 1973 y 1984, a pesar de la dictadura, aparecen 34 revistas. Cuadernos de Granaldea y Foro Literario logran editar más de cinco números. Uno, Nexo, Ficciones, Numen, Trilce, A, B y una larga lista no llegan a su número tres. Además de Maldoror como revista de significación importante aunque difusión limitada, aparece Cinemateca Revista editada por la Cinemateca Uruguaya.

IV. El restablecimiento de la democracia, en 1985 marca el comienzo del último período que llega hasta nuestros días. Además de Maldoror, aparecen Punto y Aparte, Relaciones, La Oreja Cortada, La del Taller, La Crítica, Poética, Grafias, Smog. Otras como Letras Femeninas en Uruguay, Abrelabios, Grafo, que sólo llegan a su número uno. Además de una decena de revistas marginales de circulación restringida como Basura, Detritus, Públicas Virtudes. En total suman veinte y si bien las técnicas de impresión en algunos casos llaman la atención por su disparidad (Detritus es editada en fotocopiadora color, Públicas Virtudes imprime su pliego central a Ectógrafo), el ritmo de edición-desaparición se mantiene en relación a las décadas anteriores.

Una vez más, los números descubren datos que pasan desapercibidos en lo cotidiano. Tal es el caso de nombres que se repiten como colaboradores en diversas revistas. ${ }^{7}$ Cada década tiene los suyos, pero de la misma forma en que hay revistas que abarcan tres décadas, hay nombres que más que colaboradores, son viajeros del tiempo.

Al no existir en nuestro país un Instituto de Verificación de Circulación es imposible saber con certeza los tirajes de cada revista. De todas maneras se sabe que publicaciones que hayan tirado mil ejemplares por número no superan, en

7 Rodríguez Monegal, Ángel Rama, Arturo S. Visca, Domingo Bordoli, M. Benedetti, Emilio Oribe, Juan Carlos Onetti, Martínez Carril, Martínez Moreno, Medina Vidal, Julio Da Rosa, son algunos de los nombres que se repiten como colaboradores en más de siete publicaciones diferentes. El viajero del tiempo a que hacíamos referencia es a Carlos Sabat Ercasty, colaborador en más de una docena de publicaciones. 
estos últimos cuarenta años, la cifra de 12 , siendo el promedio 650 ejemplares por número.

Queda, antes y después del número, lo que éste no pudo medir, el residuo, lo irreductible, lo que no se escribe.

MANIFIESTOS

La poesía es tan necesaria como la leche, bébala u sted y désela a sus hijos. Agamenón Castrillón

El hombre tiende a memorizar elementos trascendentes y no inmanentes, de ahí la aparición primero de la poesía y luego, en última instancia, de la prosa con su forma de discurso jurídico. Además, el hombre enmienda, transforma y metaforiza la realidad. Realidad donde está inserto lo poético, la poesía, el poema.

La poesía como lugar imaginario de todos los objetos posibles, tiene en algunas revistas casi la totalidad de su espacio. Aqui Poesía, publicación que aparece en octubre de 1962, editó a lo largo de ocho años más de cuarenta títulos. Entre ellos se encuentran libros como La hora $O$ de Ernesto Cardenal; El niño ya lo sabe de Roberto Ibáñez; Historias de las violetas de Marosa Di Giorgio; Guitarra en sombra de Clara Silva; Palabra en Vilo de Walter Ortiz y Ayala;Por modo extraño de Jorge Medina Vidal, y una serie de antologías entre las que se destacan una dedicada a la poesía checa y otra a Julio J. Casal.

Cuadernos Julio Herrera y Reissig nació a fines de 1948 como una revista donde se reunía antologías de distintos poetas. Hacia 1958 comenzó a funcionar como sello editorial, editando en menos de cuatro años más de sesenta títulos de poesía, algunos, como su número 32, seleccionados por el propio autor (Ocho poemas, de Vicente Aleixandre).

Si bien Los Huevos del Plata ${ }^{8}$ fue una revista de aparición irregular y de formato variable, a veces hasta incómoda de leer, se la recuerda porque bajo la serie La Cáscara del Huevo nos hizo conocer "Aullido y otros poemas" de Allen Ginsberg, "Gelatina" de Mario Levrero y "Mojos y canciones de protesta" de Horacio Buscaglia.

Papel de Poesía fue una publicación en el departamento de Salto, que entre los años 1953 y 1957, privilegió la crítica y el ensayo poético. Hay números dedicados a Delmira Agustini, a Juana de Ibarbourou, a María Eugenia Vaz Ferreira y Juan Zorrilla de San Martín.

Torre de los Panoramas fue el Órgano oficial de la UPU, Unión de Poetas Uruguayos [sic]. Llegó a editar cinco números, todos ellos de homenaje. El

${ }^{8}$ Editó 15 números, número cero incluido, entre los años 1965-1969. 
número cuatro, en homenaje a Pablo Neruda en su muerte, y el número cinco en homenaje a Julio Herrera y Reissig en el centenario de su nacimiento.

LaUrpila es ofue el boletíninformativo de la Casa del PoetaLatinoamericano [sic]. Bajo el nombre "Ediciones de la Urpila" se publicaron dos antologías de poetas uruguayos contemporáneos, y una decena de libros de poesía de autores desconocidos.

Poética es uno de los intentos recientes más serios de revista cultural, que le dedica un amplio espacio a la poesía. En sus páginas, y en los seis números publicados se pueden encontrar breves ensayos sobre "La polilogía de la palabra en César Vallejo"; o "La modernidad en Vicente Huidobro", textos originales, inéditos, olvidados y marginales y además una excelente muestra de poesía brasileña actual en su número 2-3; y una interesante entrevista a Eduardo Milán hecha por el también poeta Roberto Apprato, en su último número publicado.

Finalmente, hay que destacar el trabajo regular y sin pausas del grupo "Ediciones de UNO". Luego de publicar un único número de la revista UNO en la cultura, los jóvenes poetas Agamenón Castrillón, Gustavo Wojciechowski y Héctor Bardanca emprenden la actividad editorial y desde agosto de 1982 hasta diciembre de 1990 publican más de cuarenta títulos de poesía, sin descuidar el mercado de suscripciones (medio por el cual se financia la editorial)intercalando libros de cuentos, canciones, títeres, dibujos y hasta una Agenda Ilustrada de los Derechos Humanos.

En la colección de poesía, se publican tres libros de A. Castrillón (Perzonas, Perzorales y 13 instrucciones y una traición sobre el indio en la Banda Oriental); dos libros de Gustavo Wojciechowski (en)AJENA/ACCIÓN, y Segundas Impresi(ci)ones; Seis mariposas tropicales de Hugo Achugar; Fantasmas en la máquina de AtilioPérez Da Cunha; Canciones para alegrar una niña de Mauricio Rosencof; Puesto encima el corazón en llamas de Luis Bravo y Orificio de salida de Héctor Bardanca. Además, publican Introducción a César, libro con 20 poemas de Fernández Moreno y dos libros del notable poeta salvadoreño Roque Dalton, Taberna y Un libro levemente odioso.

En mayo de 1985 la editorial hizo un nuevo intento de revista que se llamó El Manojo. Su primer número estuvo dedicado a Roque Dalton y a César Fernández Moreno. En la sección reportajes, se entrevista a Mauricio Rosencof y en las páginas de poesía propiamente dicha se encuentran unos inéditos de Humberto Megget y una antología de la nueva poesía sueca. En diciembre del mismo año sale el segundo y último número dedicado a Marcel Duchamp y a Ezra Pound: "poeta, teórico, fascista, promotor".

Cualquiera que sean las ventajas de la venta a domicilio no se puede pensar seriamente en ella como un medio seguro de difusión. "Ediciones de UNO", al renunciar al mercado tradicional del libro y optar por una distribución semialternativa, limita su competitividad con las demás editoriales y se obliga a distraer su voluntad de trabajo buscando nuevos mercados. A pesar de lo 
señalado, el consejo editor insiste con la receta de sus primeros años, intentando una salvación que a esta altura de los acontecimientos parece imposible.

\section{PASIONES}

Para tratar de definir una serie de fenómenos sociales y también emocionales, un segmento de nuestra juventud (la que lee Punto y Aparte, Relaciones, La Oreja Cortada) utiliza el término post-moderno. Si se las interroga se verá que el término, para ellos, tanto sirve para propiciar desequilibrios ideológicos como para dinamizarlos, tanto para identificar una corriente arquitectónica norteamericana como para justificar estados de ánimo zigzagueantes.

Algunos estudiosos de la pretendida post-modernidad uruguaya proponen cambiar el término por el de post-post-modernidad para identificar ese mismo fenómeno, más la conciencia de tal.

Esta doble existencia de los conceptos así concebida (mental y social), invita a la reflexión, a llegar hasta el final de esta oposición que mal disimula un ambiguo movimiento dialéctico.

Tal puede ser el caso de los lectores de estas tres publicaciones que leen para reforzar sus convicciones y en donde lo escrito se manifiesta como significante cargado de prescripciones, sumergiendo al individuo y a los grupos en un contexto que proyecta un cierto orden social y mental.

Un orden social y mental donde lo único definido es la indefinición y donde la premisa básica pone de relieve tanto una crítica a todas las ideologías como un análisis de los referentes.

Un estudio de estos terremotos universales, mostraría cómo, después de cada sacudón, aparece una nueva certeza, aún más firme.

La propuesta de estas revistas es tan amplia que logra crear actitudes de adhesión, debido a que no cometen el error de "pedir" que la gente le crea. Lo único que hacen en más de una oportunidad es hacer recordar con la rapidez de un clip de video, ciertas imágenes, expresiones, citas al pie y que otras (sus posibles refutantes) se tornen relativamente inalcanzables. Por otra parte su discurso no hace que la gente crea en las instituciones y en sus valores, o que deje de creer; lo que sí logra con cierta eficacia es hacer difícil de articular o imaginar las posibles alternativas a los problemas reales. Todo esto sin mencionar hasta ahora la inclinación a una cultura alternativa de revista, donde el lector conoce unos versos, no todo el poema; capítulos de una novela, no toda la novela; lo que dicen o escriben de Octavio Paz, no a Octavio Paz; y lo que es peor aún, la metonímica creencia de algunos lectores en suponer que con la parte leída de un autor se lo comprende en su totalidad.

I. La revista literaria como objeto cultural puede tener pluralidad de verdades contradictorias. Si consideramos válida esta aseveración podemos entender sin demasiados preámbulos la retórica de Punto y Aparte. Ya en su 
primer número (julio 1987), el mismísimo presidente de la República se preguntaba en un artículo “¿Dónde está la seguridad de los Derechos Humanos?" A lo largo de 37 números (octubre/1990) pasaron el hoy presidente Lacalle -en ese entonces, Senador - haciendo un aburrido análisis del sistema educativo nacional (no. 4, octubre/87) hasta el Sr. George Bush (en ese entonces vicepresidente de Estados Unidos) justificando "la paz mediante la fortaleza" (no. 15 agosto/88). Eso en lo que atañe a la visión política nacional e internacional; porque en su casi centenar de páginas se encontraron los nombres de Anthony Burgess, Umberto Eco, Jean Baudrillard, Octavio Paz, Alain Touraine, Edgar Morin, Italo Calvino, Salman Rushdie, García Márquez, Juan Carlos Onetti, y hasta un cuento inédito (jcuándo no!) de Ernest Hemingway. Entre sus colaboradores nacionales figuraron Gustavo Escanlar, Héctor Bardanca, Elvio Gandolfo, Rafael Courtoisie, Luciano Alvarez, José Luis Castagnola, Alvaro Díez de Medina.

En el número 20 se le dedicó amplio espacio a "el arte de narrar". Figuran con toda justicia Héctor Galmés, Anderson Banchero y Mario Levrero.

La publicación contó también con una lujosa presentación, una diagramación impecable $y$, justo es decirlo, con un nunca declarado apoyo semi-oficial. Además logró captar un importante número de anunciantes económicamente muy fuertes, que le permitió incluso promocionarse en televisión con un original corto publicitario.

Semi-oficial, post-moderna, complaciente, plural, competitiva, contestataria, polémica; lo cierto es que el número 37 marcó el final de una publicación que se ganó un lugar entre las mejores revistas culturales uruguayas.

II. El mundo se divide en actos de habla y actos de dicción. Estos últimos son aquéllos que construimos, con la intencionalidad de trascender nuestro entorno. Por un problema de madurez, hay actos de dicción que se especializan, lo que en cierta manera es una forma de incapacidad. Esto tal vez sea lo que le sucede a Relaciones. Su primer número salió a la venta en junio de 1984 y en febrero de 1991 llevaba publicado su número 81 . Sus temas son: la comunicación y la significación, los derechos lingüísticos del niño, los límites del lenguaje, semiótica literaria, la metáfora, semiótica de la ropa interior, lugares de la locura, psicoanálisis, violencia, fenomenología del elitismo, los intelectuales, el exilio y el poder, subdesarrollo, drogadicción, el lugar del sujeto en las ciencias sociales.

III. La decepción y la frustración de los jóvenes uruguayos es inmensa. Y esto no cambiará mientras a su apetito por todo, se lo intente calmar con signos. Como resultado, los jóvenes agrupan. Protestan. Contestan, no sin antes intentar sacar provecho de la situación. Es más significativo el rechazo presentado por grupos que adhieren (de alguna forma hay que decirlo) a publicaciones comoLa Oreja Cortada. Publicación que desde su primer número 
intentó escandalizar la reciente establecida democracia (1987) posando desnudos y de cuerpo entero todos los integrantes de su Consejo Editor. Sabido es que la camaradería erótica va contra los principios estéticos, y que en el Uruguay existe una lista de prioridades para escandalizarse en donde no figura esta foto. De todos modos si la intención era salirse del código para intentar provocar afasia o sorpresa, ahora ya saben que también movió a dudosas risas.

Y si bien la primera sensación que se tenía de ella era la de una lectura libertina, existía en casi todas sus páginas una propuesta literaria seria, con espacio para todos sus géneros.

Para leer el segundo número, hubo que esperar tres años. El consejo editor cambió su nombre por el de "Cómplices" y el de sus colaboradores por el de "Aliados". El formato fue otro, la diagramación distinta, los tipos diferentes, pero el metadiscurso ideológico que domina a la revista fue el mismo del primer número. Este metadiscurso produce la ilusión de no envejecer, intenta reducir lo temporal en lo simultáneo, y aprovecha para negar la historia, el pasado, y dudar del futuro. Es el mismo metadiscurso que obliga a los integrantes de la revista a no perder la originalidad, en ningún detalle. Y si el primer número parecía bueno, éste lo es. En su interior nos encontramos con textos de John Lennon y Eduardo Mateo. Hay una ingeniosa solución a "La Salve Multiforme" de Francisco Acuña de Figueroa y una entrevista sin desperdicio al viejísimo Dr. Rodolfo Tálice hablando de hongos alucinógenos, además de espacio para poesía, recetas de cocina, algunas posturas malvadas, otras que lindan con la chabacanería y otras con el delirio. En este segundo número espacial (así se llama) se nota la marcada presencia de Héctor Bardanca como editor, diagramador, autor y hasta distribuidor de los 1.000 ejemplares editadosagotados.

IV. Así como se identifica a Punto y Aparte y Relaciones como publicaciones formales con una red de distribución en todo el país; así como se identifica a $L a$ Oreja Cortada como una publicación independiente, con una distribución alternativa; así también hay lugar para una tercera categoría de revistas: las marginales. Forman parte de ese segmento de realidad que no se promociona, pero que existe. Algunas de ellas no llegan a editar más de 100 ejemplares. Tal es el caso de Detritus, aparecido en enero de este año. En su editorial afirma en un tono que a cada frase se torna más hiriente y depresivo:

... todo lo que nos sucede es adorable ... disfrutemos porque estamos asistiendo a uno de los momentos más estelares de la humanidad ... acostumbrémonos a quitarle trascendencia a la emoción. Acostumbrémonos al insulto ... aceptemos ser la última forma del amor: la agresión ....9

\footnotetext{
${ }^{9}$ Plebiscito de abril de 1989 donde se intentó derogar la Ley de Caducidad, que exime de toda culpa a aquellos integrantes de nuestras Fuerzas Armadas acusados de violar los derechos humanos.
} 
Hay diez páginas con poemas de César Vallejo, Pablo Neruda, Juan José Tablada, Oliverio Girondo y el soneto "Oblación Abracadabra" de Julio Herrera y Reissig. Hacia el final de la revista hay un artículo firmado por Aurelio del Hebrón III ${ }^{10}$ titulado: "El amor es siempre una disponibilidad" que sirve de prólogo a la brillante traducción de "El entierro de los muertos", primera parte de The Waste Land de Thomas Stearns Eliot.

\section{CONCLUSIONES}

Todo hecho literario para su estudio supone una tríada compuesta por escritores, libros y lectores. La revista literaria como obra en sí misma o como promoción de otras obras, forma parte de una compleja trama editorial (en la mayoría de sus casos) que dificulta su estudio. Se acostumbra decir de ella que surge como deseo incontrolado de un grupo de jóvenes escritores que no accede todavía al libro. Por esta razón, la publicación no será conocida más allá del medio local, no conquistará un público numeroso y sólo será leída por lectores iniciados o del ambiente literario.

Lo que generalmente se nos olvida decir es que, gracias a ella, la literatura se ha liberado de su aire sacramental, accediendo el lector al conocimiento de la obra y al reconocimiento de sí mismo en la reflexión, del otro en el concepto, y del ser en general, en la certeza de que el hombre no se agota en una verdad única.

Finalmente recordar que la revista literaria, en tanto texto, no se consume en lo que enuncia, sino en lo que desencadena en la realidad.

\section{BIBLIOGRAFIA}

Barite, M. y Ceretta M., Guía de revistas culturales uruguayas 1895-1985 (Montevideo: Ediciones "El Galeón", 1989).

Baudrilliard, J., La economía política del signo (Buenos Aires: Siglo XXI, 1984). Block de Behar, L., Una retórica del silencio (México: Siglo XXI, 1984).

Escarpit, Roberto L., Sociología de la literatura (Buenos Aires: Los libros del Mirasol, 1962).

Rama, Ángel, La generación crítica (Montevideo: Arca, 1972).

Rodríguez Monegal, Emir, Literatura uruguaya de medio siglo (Montevideo: Alfa, 1966).

Sambarino, Mario, La cultura nacional como problema (Montevideo, Nuestra Tierra, 1969).

Schudson, Michael,Advertising, the Uneasy Persuassion (California:Basic Books, 1985).

${ }^{10}$ Aurelio del Hebrón era el seudonimo utilizado por Alberto Zum Felde, a principios de siglo. 
Revistas consultadas:

$A$ - Número único (1979)

Abrelabios - Número único (1984)

Alfar - Nos. 45; 76; 90 (1923-1955)

Antifaz - Número único (1990)

Aquí Poesía - 12; 38; 39; 40 (1962-1970)

Asir - Colección completa (1948-1959)

$B$ - Número único (1980)

Cinemateca Revista - Colección completa (1977-H)

Cuadernos de Granaldea - Nos. 1; 2; 3 (1980-1984)

Detritus - Número único (1991)

Grafo - Número único (1985)

La Crítica - Nos. 1; 2; 3 (1985)

La del Taller - 1; 2 (1984-1985)

La Licorne - 9; 16 (1947-1961)

La Oreja Cortada - 1; 2 (1987-H)

La Urpila - 1; 43 (1980-1985)

Los Huevos del Plata - 1; 4; 5 (1965-1969)

Maldoror - 12; 16; 17; 18; 19; 20; 21 (1967-1985)

MonteSexto - Número único (1969)

Nexo - Nos. 1 y 2 (1975-1976)

Número - 3 y 4 (1949-1964)

Poética - $1 ; 2 ; 3 ; 4 ; 5$ y 6 (1985-1986)

Públicas Virtudes - Número único (1990)

Puente - Número único (1963)

Punto y Aparte - Colección completa (1987-1990)

Relaciones - Colección completa (1984-H)

Uno en la cultura - Número único (1982)

Agradecimientos: este trabajo no se hubiera logrado sin la paciente e invalorable colaboración del Sr. Arturo Sergio Visca. 
